\title{
Immune checkpoint inhibitor-associated thyroid dysfunction: a disproportionality analysis using the WHO Adverse Drug Reaction Database, VigiBase
}

\section{Xuefeng Bai*, Xiaoyu Chen*, Xiaohong Wu, Yinqiong Huang, Yong Zhuang and Xiahong Lin}

Department of Endocrinology, The Second Affiliated Hospital of Fujian Medical University, Quanzhou City, Fujian Province, China

*(X Bai and X Chen contributed equally to this work)

\begin{abstract}
Objective: Our study aimed to identify and characterize thyroid dysfunctions associated with immune checkpoint inhibitors (ICls).

Design: Data were obtained from VigiBase, between January 1, 2011 to March 6, 2019.

Methods: All thyroid drug-adverse events are classified by group queries according to the Medical Dictionary for Regulatory Activities. Information component (IC) and reporting odds ratio (ROR) were considered as measures of disproportionality for the assessment of association between ICIs and thyroid dysfunctions. We used IC to identify meaningful drug-adverse events while using ROR to compare differences in the reporting of drug-adverse events caused by different ICI subgroups. Positive IC values are deemed significant.

Results: Compared with the full database, the following $\mathrm{ICl}$-associated thyroid dysfunctions were over-reported: hypothyroidism (1125 reports for ICIs vs 12495 for all drugs; Information Component 4.28 (95\% Cl: 4.18-4.35)), hyperthyroidism (926 vs 7538; 4.66 (95\% Cl: 4.55-4.74)), thyroiditis (294 vs 1237; 5.40 (95\% Cl: 5.21-5.54)), thyrotoxic crisis (11 vs 288; 3.55 (95\% Cl: 2.61-4.20)). Hypothyroidism was over-reported for patients treated with ICI combination therapy versus those treated with ICI monotherapy (ROR 1.3 (95\% Cl: 1.1-1.7)), and the same was observed for hyperthyroidism (ROR: 1.9 (95\% Cl: 1.5-2.4)), thyroiditis (ROR: 3.3 (95\% Cl: 2.3-4.8)), thyrotoxic crisis (ROR: 11.5 (95\% $\mathrm{Cl}$ : 2.4-53.8)). All 11 thyrotoxic crisis cases were malignant melanoma patients, of which seven occurred under ICI combination therapy.

Conclusions: Thyroid dysfunction may occur after ICI therapies, and severe thyrotoxic crisis may even occur. Raising awareness of $\mathrm{ICl}$-associated thyroid dysfunction can improve the detection and treatment of these diseases.
\end{abstract}

\section{Introduction}

Cancer immunotherapy has a long and tortuous history, and immune checkpoint inhibitors (ICIs) are considered to be the most successful immunotherapy to date, significantly improving the survival rate of some advanced cancers, especially metastatic melanoma and lung cancer (1). In recent years, several ICIs for malignant cancer therapy have received FDA approval for their $\mathrm{T}$ cell activation and anti-tumour activities, including antiCTLA-4 mAbs (ipilimumab), anti-PD-1 mAbs (nivolumab and pembrolizumab) and anti-PD-L1 mAbs (atezolizumab

Published by Bioscientifica Ltd. 
and durvalumab). However, these therapies may also induce a large series of autoimmune adverse events, which are known as immune-related adverse events (irAEs), particularly endocrine irAEs, including thyroid dysfunction, hypophysitis, primary adrenal insufficiency, and type 1 diabetes (2). In the past 3 years, reports of ICI-TD have increased, including temporary thyrotoxicosis, temporary or long-term hypothyroidism, thyroiditis $(3,4,5)$, and occasionally severe thyrotoxic crisis $(6,7)$. Although the severity of most irAEs is generally grade $1-2$ $(8,9)$, life-threatening complications may occur if not detected and treated in time.

Cancer immunotherapy has only appeared in recent years, and physicians with clinical experience in the diagnosis and management of immunotoxicity are often those who actively participate in clinical trials. Therefore, many clinical oncologists and endocrinologists are still unfamiliar with these ICI-TDs. In addition, previous scientific literature lacks reports on the complete spectrum and clinical characteristics of ICI-TD, especially case reports of thyrotoxic crisis. In this study, we use VigiBase, the WHO global database of individual case safety reports (ICSRs) (10), which is managed by the Uppsala Monitoring Centre (UMC) in Sweden, to fully understand the spectrum and clinical characteristics of ICI-TD.

\section{Methods}

\section{Study data source}

In this study, we obtained data from VigiBase. VigiBase includes reports from more than 130 countries, represents over $90 \%$ of the world's population, and contains more than 19 million reports of suspected adverse drug reactions, submitted by member countries of the WHO Programme for International Drug Monitoring (WHO PIDM) since 1968. These adverse reactions contain details on administrative data, patient data, adverse drug reactions (ADRs) data, medication data, and additional information obtained from different sources, such as physicians, pharmacists, other health care professionals, and patients. In VigiBase, ADRs are coded according to the Adverse Reaction Terminology (WHO-ART) and Medical Dictionary for Regulatory Activities (MedDRA; www. who-umc.org).

\section{Design and definition}

This is an observational, retrospective, and disproportionality analysis based on the VigiBase database, reporting suspected thyroid irAEs associated with ICIs from January 1, 2011 to March 6, 2019 and describing the clinical characteristics of these cases in detail. The reference group is all adverse reactions in the VigiBase database during the same study period. It should be noted that the reported drugs are encoded according to WHODrug. The five ICI drugs reported in this study are anti-CTLA-4 mAbs (ipilimumab), anti-PD-1 mAbs (nivolumab and pembrolizumab) and anti-PD-L1 mAbs (atezolizumab and durvalumab). Additionally, MedDRA's preferred term level (version 20.1) was used in this study to specify suspected ADRs. The definition of hypothyroidism includes the following MedDRA terms: hypothyroidism, autoimmune hypothyroidism, and primary hypothyroidism. The definition of thyroiditis includes the following MedDRA terms: thyroiditis, silent thyroiditis, acute thyroiditis, subacute thyroiditis, chronic thyroiditis, and infectious thyroiditis. Other thyroid-related MedDRA terms include hyperthyroidism, thyrotoxic crisis, autoimmune thyroid disorder, thyroid disorder, secondary hypothyroidism, thyroid mass, and so forth. (Table 1).

For each adverse reaction associated with ICI therapies, we obtained the following: management information (report ID, region of origin, date of reporting, and notifier), patient characteristics (sex and age group), drug information (WHODrug name, reason for drug use, and dosage regimen), and ADR information (report type, MedDRA classification terms, time to onset of ADRs, and final outcomes). A fatal outcome was defined as causing death; being lifethreatening; requiring hospitalization (initial or prolonged); or causing any other medically important diseases. In addition, the dosage regimen includes the administered dose (reported in $\mathrm{mg} / \mathrm{kg}$ or single total dose), the route of administration, and the frequency of dosage. It is worth noting that adverse drug reactions of this study began on January 1, 2011, because the first ICI drug (Ipilimumab) was approved by the FDA in 2011, and the number of adverse reactions from 2008 (the first ICI adverse drug reaction) to 2011 was too small to analyze (only three cases, one each for hypothyroidism, diarrhea and hepatitis).

\section{Statistical analysis}

As a spontaneous reporting system, the VigiBase database allows for disproportionality analysis, which we used to evaluate whether the occurrences of thyroid irAEs were differentially reported between ICIs and other drugs in the full database. We also used disproportionality analysis to compare thyroid irAEs among different ICI regimens: anti-PD-1 or anti-PD-L1 monotherapy versus anti-CTLA-4 
Table 1 ICls-associated thyroid irAE reports versus those reported in the full database from VigiBase (from January 1, 2011 to March 6, 2019). ICSRs are presented as $\mathrm{n}(\%)$. Information component (IC) reflects a strength of association between the drugadverse event. Positive IC values are thresholds for signal detection with VigiBase. ICls include Ipilimumab, Nivolumab, Pembrolizumab, Atezolizumab, Durvalumab and combination therapy (Ipilimumab plus Nivolumab). All thyroid irAEs classified by group queries according to the Medical Dictionary for Regulatory Activities (MedDRA, version 20.1).

\begin{tabular}{|c|c|c|c|}
\hline & $\begin{array}{c}\text { ICSRs for ICIs } \\
\text { (total ICls reports: 61931) }\end{array}$ & $\begin{array}{l}\text { ICSRs in full database } \\
\text { (total reports: 13300773) }\end{array}$ & IC $(95 \% \mathrm{Cl})$ \\
\hline Hypothyroidism* & $1125(1.82 \%)$ & $12495(0.09 \%)$ & $4.28(4.18-4.35)$ \\
\hline Hyperthyroidism\# & $926(1.50 \%)$ & $7538(0.06 \%)$ & $4.66(4.55-4.74)$ \\
\hline Thyroiditis & $294(0.47 \%)$ & $1237(0.01 \%)$ & $5.40(5.21-5.54)$ \\
\hline Thyrotoxic crisis & $11(0.02 \%)$ & $288(<0.01 \%)$ & $3.55(2.61-4.20)$ \\
\hline Autoimmune thyroid disorder & $159(0.26 \%)$ & $1535(0.01 \%)$ & $4.37(4.11-4.56)$ \\
\hline Thyroid disorder & $172(0.28 \%)$ & $5756(0.04 \%)$ & $2.76(2.51-2.94)$ \\
\hline Anti-thyroid antibody positive & $8(0.01 \%)$ & $514(<0.01 \%)$ & $1.93(0.72-2.74)$ \\
\hline
\end{tabular}

*The definition of hypothyroidism includes the following MedDRA terms: hypothyroidism ( $n=1125)$, autoimmune hypothyroidism ( $n=37)$, primary hypothyroidism $(n=11)$. "The definition of thyroiditis includes the following MedDRA terms: thyroiditis $(n=266)$, silent thyroiditis $(n=12)$, acute thyroiditis $(n=11)$, subacute thyroiditis $(n=4)$, chronic thyroiditis $(n=2)$, infectious thyroiditis $(n=1)$. ICl-associated thyroid diseases also include some with negative IC values, such as thyroid mass $(n=5)$, post procedural hypothyroidism $(n=2)$, medullary thyroid cancer $(n=1)$, metastases to thyroid $(n=1)$, secondary hyperthyroidism $(n=1)$.

ICIs, immune checkpoint inhibitors; ICSRs, individual case safety reports.

monotherapy, as well as combination therapy (anti-CTLA-4 plus anti-PD-1 or anti-PD-L1) versus monotherapy. The analyses are based on observed reporting relationships and aim to identify drug-adverse event associations with a relative frequency that differs from the overall reporting in the database. Disproportionality analysis compares the proportion of selected specific ADRs reported for a single drug or group of drugs (e.g., ICIs) with the proportion of the same ADRs for a control group of drugs (e.g., complete database). These methods use total number of adverse drug reactions for each group of drugs as a surrogate denominator.

Disproportionality can be calculated by either the information component (IC) or the reporting odds ratio (ROR). However, compared to the reporting odds ratio, information component can provide a conservative measure of association and reduce the risk of highlighting spurious associations, in particular for events with very low expected frequencies in a large database (e.g., VigiBase) (11). Information component values have only been proven for comparison of drug-adverse events versus the full database and cannot be used to compare disproportionate reporting among different ICI therapies. Therefore, we used information components to identify meaningful drug-adverse event signals while using the reporting odds ratio to compare differences in the reporting of adverse drug reactions caused by different drug subgroups (e.g., different ICIs).

Calculation of the IC derived from a Bayesian confidence propagation neural network (BCPNN) is the first to be used for worldwide drug signal monitoring by the Uppsala Monitoring System, which reflects the strength of the drug-adverse event association. The strength is expressed by the logarithm of the ratio of the prior probability and the posterior probability of ADR occurrence; the statistical formula is described elsewhere and detailed in the attachment (Attachment 1). A positive information component value indicates that a particular drug-adverse event pair has been reported more often than expected, based on all the reports in the database. The $95 \%$ confidence interval provides information on the stability of information component values: the narrower the interval, the higher the stability $(12,13)$.

Disproportionality for thyroid irAE reports induced by different ICI regimens was estimated by calculating the reporting odds ratio $(95 \% \mathrm{CI})$. The ratio of target adverse events to non-target adverse events reflects a significant imbalance if the lower bound of the confidential interval is larger than 1 , indicating that the adverse reactions of the drug are notable ADR signals. Comparisons between different drug regimens were performed using the Bonferroni adjustment method, and the ROR confidence interval was adjusted using $P=0.01$. Descriptive statistical methods were used to summarize clinical characteristics of adverse drug reactions. One-way ANOVA was used to compare quantitative variables. The $\chi^{2}$ test was used to compare qualitative variables.

\section{Results}

From January 1, 2011 to March 6, 2019, there were 61,931 adverse events reported in patients who received ICIs 
and 13,300,773 adverse events reported in patients who received any drugs (full database) in VigiBase. Table 1 shows information component values for various ICI-TDs compared to the same adverse event in the full database: ICI-associated hypothyroidism (1125 reports for ICIs vs 12495 for the full database; Information Component 4.28 (95\% CI: 4.18-4.35)); ICI-associated hyperthyroidism (926 reports for ICIs vs 7538 for the full database; Information Component 4.66 (95\% CI: 4.55-4.74)); and ICI-associated thyroiditis (294 reports for ICIs vs 1237 for the full database; Information Component 5.40 (95\% CI: 5.21-5.54)). It is worth noting that we also found 11 cases $(0.04 \%)$ of rarely reported ICI-associated thyrotoxic crisis (288 reports for the full database; Information Component 3.55 (95\% CI: 2.61-4.20)). For the above four ICI-TDs, all information component values are positive, and the $95 \%$ CIs are narrow, which indicates that these adverse events are significantly associated with ICI therapies. We mainly focus on these four ICI-TDs (total of 2356 cases, 2147 reports from medical institutions and 209 cases from nonmedical) to report differences in various ICI subgroups and to describe the clinical characteristics of each ICI-TD. In addition, reports of irAEs such as autoimmune thyroid disorder, thyroid disorder, and anti-thyroid antibody positivity have also increased. However, due to the lack of inspection reports for these adverse drug reactions, there is no way to further determine the etiology; thus, these adverse events are not reported in detail in this study.

Compared with the full database, ROR values of these four ICI-TDs with different ICI treatments were significantly different and the highest for combination therapies. Further analysis found that the four thyroid irAEs were over-reported for patients who received ICI combination therapy versus those who received ICI monotherapy: hypothyroidism (ROR: 1.3 (95\% CI: 1.11.7)), hyperthyroidism (ROR: 1.9 (95\% CI: 1.5-2.4)), thyroiditis (ROR: 3.3 (95\% CI: 2.3-4.8)), and thyrotoxic crisis (ROR: 11.5 (95\% CI: 2.4-53.8)). Hypothyroidism, hyperthyroidism, and thyroiditis were over-reported for patients who received anti-PD monotherapy versus those who received anti-CTLA4 monotherapy (ROR: 2.4 (95\% CI: 1.8-3.1); ROR: 3.6 (95\% CI: 2.5-5.3) ROR: 1.9 (95\% CI:1.1-3.2)), except for thyrotoxic crisis (Table 2).

The reports for ICI-associated hypothyroidism, hyperthyroidism, and thyroiditis were mainly from Europe (711/1125 (63.20\%), 474/926 (51.19\%), 160/294 $(54.42 \%)$, respectively), while 5 of all 11 thyrotoxic crisis cases were from the United States. Among all the analyzed ICI-TDs, there were more male cases than female cases, but without statistical significance (hypothyroidism 54.99 vs $45.01 \%$; hyperthyroidism 58.19 vs $41.81 \%$; thyroiditis 59.85 vs $40.15 \%$; thyrotoxic crisis 54.55 vs $45.45 \% ; \chi^{2}$ test for overall subgroup comparison, $P=0.369$ ). Table 3 shows that reports of hypothyroidism, hyperthyroidism, and thyroiditis were observed mainly in patients with lung cancer and malignant melanoma, while all 11 cases of thyrotoxic crisis were reported to have melanoma.

Compared with other ICIs, nivolumab-associated hypothyroidism (578 cases, 51.38\%), hyperthyroidism (447 cases, $48.27 \%$ ) and thyroiditis (128 cases, $43.54 \%$ ) had the most reports. More importantly, thyrotoxic crisis was reported mainly under ICI combination

Table 2 Major ICl-associated thyroid dysfunction compared to full database from VigiBase (January 1, 2011 to March 6, 2019). Anti-CTLA-4 refers to any ICSR treated with Ipilimumab. Anti-PD-1/PD-L1 refers to any ICSR treated with any of the following four drugs: Nivolumab, Pembrolizumab, Atezolizumab, Durvalumab. ICI Combination refers to any ICSR treated with anti-CTLA-4 (Ipilimumab) in combination with any one of the anti-PD-1/PD-L1. Comparison between different drug regimens were performed using the Bonferroni adjustment method; thus, the ROR confidence intervals were calculated using P=0.01 to adjust.

\begin{tabular}{|c|c|c|c|c|c|}
\hline & $\begin{array}{l}\text { Anti-CTLA-4 vs } \\
\text { full database }\end{array}$ & $\begin{array}{l}\text { Anti-PD-1/PD-L1 vs } \\
\text { full database }\end{array}$ & $\begin{array}{l}\text { ICI combination vs } \\
\text { full database }\end{array}$ & $\begin{array}{c}\text { Aanti-PD-1/PD-L1 vs } \\
\text { anti-CTLA-4 }\end{array}$ & $\begin{array}{l}\text { ICI combination vs } \\
\text { monotherapy }\end{array}$ \\
\hline \multicolumn{6}{|l|}{ Hypothyroidism } \\
\hline $\mathrm{N} / \mathrm{N}$ & $84 / 12495$ & $922 / 12495$ & $119 / 12495$ & $922 / 84$ & 119/1006 \\
\hline ROR $(95 \% \mathrm{Cl})$ & $10.1(8.2-12.4)$ & $23.7(22.1-25.3)$ & $28.3(23.7-33.8)$ & $2.4(1.8-3.1)$ & $1.3(1.1-1.7)$ \\
\hline \multicolumn{6}{|l|}{ Hyperthyroidism } \\
\hline $\mathrm{N} / \mathrm{N}$ & $44 / 7538$ & $751 / 7538$ & $131 / 7538$ & $751 / 44$ & 131/795 \\
\hline ROR $(95 \% \mathrm{Cl})$ & $8.7(6.5-11.6)$ & $31.3(29.0-33.8)$ & $51.4(43.3-61.0)$ & $3.6(2.5-5.3)$ & $1.9(1.5-2.4)$ \\
\hline \multicolumn{6}{|l|}{ Thyroiditis } \\
\hline $\mathrm{N} / \mathrm{N}$ & $24 / 1237$ & 207/1237 & $63 / 1237$ & $207 / 24$ & $63 / 231$ \\
\hline ROR $(95 \% \mathrm{CI})$ & $34.1(22.8-51.2)$ & $56.8(48.9-66.0)$ & $186.2(145.5-238.5)$ & $1.9(1.1-3.2)$ & $3.3(2.3-4.8)$ \\
\hline \multicolumn{6}{|l|}{ Thyrotoxic crisis } \\
\hline $\mathrm{N} / \mathrm{N}$ & $2 / 288$ & $2 / 288$ & $7 / 288$ & $2 / 2$ & $7 / 4$ \\
\hline ROR $(95 \% \mathrm{Cl})$ & $9.6(2.4-38.6)$ & $6.4(1.6-25.7)$ & $87.9(45.3-170.8)$ & $0.7(0.1-8.7)$ & $11.5(2.4-53.8)$ \\
\hline
\end{tabular}

https://eje.bioscientifica.com 
Table 3 Basic information with ICl-associated thyroid dysfunction cases reported in VigiBase (January 1, 2011 to March 6, 2019 ). Data are presented as as $n(\%)$.

\begin{tabular}{|c|c|c|c|c|}
\hline & Hypothyroidism $(n=1125)$ & Hyperthyroidism $(n=926)$ & Thyroiditis $(n=294)$ & $\begin{array}{l}\text { Thyrotoxic crisis } \\
\quad(n=11)\end{array}$ \\
\hline \multicolumn{5}{|l|}{ Year } \\
\hline 2011 & $1(0.09 \%)$ & $1(0.11 \%)$ & 0 & 0 \\
\hline 2012 & $11(0.98 \%)$ & $6(0.65 \%)$ & $1(0.34 \%)$ & 0 \\
\hline 2013 & $6(0.53 \%)$ & $5(0.54 \%)$ & $1(0.34 \%)$ & 0 \\
\hline 2014 & $11(0.98 \%)$ & $13(1.4 \%)$ & $2(0.68 \%)$ & 0 \\
\hline 2015 & $50(4.44 \%)$ & $26(2.81 \%)$ & $6(2.04 \%)$ & $1(9.09 \%)$ \\
\hline 2016 & $135(12.0 \%)$ & $84(9.07 \%)$ & $38(12.93 \%)$ & $1(9.09 \%)$ \\
\hline 2017 & $307(27.29 \%)$ & $237(25.59 \%)$ & $100(34.01 \%)$ & $2(18.18 \%)$ \\
\hline 2018 & $516(45.87 \%)$ & $462(49.89 \%)$ & $117(39.8 \%)$ & $5(45.45 \%)$ \\
\hline 2019 & $88(7.82 \%)$ & $92(9.94 \%)$ & $29(9.86 \%)$ & $2(18.18 \%)$ \\
\hline \multicolumn{5}{|l|}{ Gender } \\
\hline Female & 474/1053 (45.01\%) & $370 / 885$ (41.81\%) & $108 / 269(40.15 \%)$ & $5 / 11(45.45 \%)$ \\
\hline Male & $579 / 1053$ (54.99\%) & $515 / 885(58.19 \%)$ & $161 / 269(59.85 \%)$ & $6 / 11(54.55 \%)$ \\
\hline \multicolumn{5}{|l|}{ Age group } \\
\hline $0-18$ & $2 / 777(0.26 \%)$ & $1 / 724(0.14 \%)$ & $1 / 213(0.47 \%)$ & 0 \\
\hline $18-44$ & 76/777 (9.78\%) & 65/724 (8.98\%) & $19 / 213(8.92 \%)$ & $1 / 8(12.5 \%)$ \\
\hline $45-64$ & $315 / 777$ (40.54\%) & $330 / 724(45.58 \%)$ & $95 / 213(44.6 \%)$ & $3 / 8(37.5 \%)$ \\
\hline More than 65 & $384 / 777$ (49.42\%) & $328 / 724$ (45.3\%) & $98 / 213(46.01 \%)$ & $4 / 8(50.0 \%)$ \\
\hline \multicolumn{5}{|l|}{ Region } \\
\hline Americas & $278(24.71 \%)$ & $146(15.77 \%)$ & $68(23.13 \%)$ & $5(45.45 \%)$ \\
\hline Asia & $125(11.11 \%)$ & $292(31.53 \%)$ & 57 (19.39\%) & $3(27.27 \%)$ \\
\hline Europe & $711(63.20 \%)$ & 474 (51.19\%) & $160(54.42 \%)$ & $3(27.27 \%)$ \\
\hline Oceania & $11(0.98 \%)$ & $14(1.51 \%)$ & $9(3.06 \%)$ & 0 \\
\hline \multicolumn{5}{|l|}{ Notifier* } \\
\hline Notifier1 & $998(88.71 \%)$ & 865 (93.41\%) & 275 (93.54\%) & $9(81.82 \%)$ \\
\hline Notifier2 & $127(11.29 \%)$ & $61(6.59 \%)$ & $19(6.46 \%)$ & $2(18.18 \%)$ \\
\hline \multicolumn{5}{|l|}{ Indication" } \\
\hline Lung cancer & 452/1017 (44.44\%) & 413/844 (48.93\%) & $108 / 261(41.38 \%)$ & 0 \\
\hline $\begin{array}{l}\text { Malignant } \\
\text { melanoma }\end{array}$ & $348 / 1017$ (34.22\%) & 269/844 (31.87\%) & $105 / 261(40.23 \%)$ & $11 / 11(100 \%)$ \\
\hline Renal cell carcinoma & $57 / 1017(5.60 \%)$ & $37 / 844(4.38 \%)$ & $15 / 261(5.75 \%)$ & 0 \\
\hline $\begin{array}{l}\text { Cancer of head and } \\
\text { neck }\end{array}$ & $28 / 1017(2.75 \%)$ & $14 / 844(1.66 \%)$ & 0 & 0 \\
\hline Urothelial carcinoma & $15 / 1017(1.47 \%)$ & $11 / 844(1.30 \%)$ & $1 / 261(0.38 \%)$ & 0 \\
\hline Lymphoma & $20 / 1017(1.97 \%)$ & $10 / 844(1.18 \%)$ & $4 / 261(1.53 \%)$ & 0 \\
\hline
\end{tabular}

*Notifier1 refers to physician, pharmacist and other health professional. Notifier2 refers to lawyer, consumer. \#Indication refers to the primary tumors treated by ICls, and the complete indications are provided in attachment 2.

therapy (ipilimumab plus nivolumab, 7 of the total 11 cases). There were a few reports of anti-PD-L1-associated thyroid dysfunction (63 cases of atezolizumab, 79 cases of durvalumab), while the spectrum of thyroid irAEs was similar to that of anti-PD-1, mainly demonstrating hypothyroidism and hyperthyroidism but not any thyrotoxic crisis cases (Table 4).

These thyroid irAE cases were monitored after the first use of ICI. Compared with anti-PD monotherapy and anti-CTLA- 4 monotherapy, the time to onset of hypothyroidism associated with ICI combination therapy was earlier (anti-PD 92 days (IQR: 53-146); anti-CTLA-4 63 days (IQR: 42-70); combination 46 days (IQR: 23-87); one-way ANOVA for overall subgroup comparison, $P<0.0001)$. However, the time to onset of hyperthyroidism and thyroiditis was not statistically different between the different ICI subgroups (one-way ANOVA for overall subgroup comparison, $P=0.990$ and $P=0.639$, respectively; Fig. 1 ).

There were reports of 136 cases of hyperthyroidism followed by hypothyroidism, also referred to as transient thyrotoxicosis. We found that thyrotoxicosis lasted for a short time with a median time of 35.5 days (IQR: 9-62.55). In addition, it is important to note that among all 11 cases of thyrotoxic crisis, only 4 cases were reported for time to onset, 2 cases were patients treated with ICI combination therapy (nivolumab plus ipilimumab), with time to onset of 7 days and 14 days, respectively, and the other 2 cases 
Table 4 Comparison of ICI regimen for thyroid dysfunction diseases reported by VigiBase (January 1, 2011 to March 6, 2019).

Data are presented as $\mathrm{n}(\%)$.

\begin{tabular}{l}
\hline \\
Drug Group \\
Nivolumab \\
Pembrolizumab \\
Atezolizumab \\
Durvalumab \\
Ipilimumab \\
Combination (Ipilimumab \\
plus Nivolumab) \\
Drug doses \\
Monotherapy with Nivolumab \\
$1-2$ mg/kg \\
$\geq 3$ mg/kg \\
$\leq 240$ mg \\
$>240$ mg to $\leq 480 \mathrm{mg}$ \\
Monotherapy with Pembrolizumab \\
2 mg/kg \\
6 mg/kg \\
$\leq 200$ mg \\
$>200$ mg \\
Monotherapy with Ipilimumab \\
3 mg/kg \\
10 mg/kg \\
$\leq 500$ mg \\
$>500$ mg \\
Combination therapy \\
Ipilimumab ( 3 mg/kg) plus \\
Nivolumab ( $1-2 \mathrm{mg} / \mathrm{kg})$ \\
Ipilimumab ( $3 \mathrm{mg} / \mathrm{kg}$ ) plus \\
Nivolumab ( $\geq 3 \mathrm{mg} / \mathrm{kg})$ \\
Ipilimumab ( $\leq 500 \mathrm{mg}$ ) plus \\
Nivolumab ( $\leq 240 \mathrm{mg})$ \\
Nilimumab \\
Natal \\
Not recovered/not resolved \\
Recovered/resolved \\
\hline \\
\end{tabular}

\begin{tabular}{c}
\hline $\begin{array}{c}\text { Hypothyroidism } \\
(n=1125)\end{array}$ \\
\hline \\
$578(51.38 \%)$ \\
$275(24.44 \%)$ \\
$34(3.02 \%)$ \\
$35(3.11 \%)$ \\
$84(7.47 \%)$ \\
$119(10.58 \%)$
\end{tabular}

\begin{tabular}{c}
\hline $\begin{array}{c}\text { Hyperthyroidism } \\
(n=926)\end{array}$ \\
\hline \\
$447(48.27 \%)$ \\
$242(26.13 \%)$ \\
$24(2.59 \%)$ \\
$38(4.1 \%)$ \\
$44(4.75 \%)$ \\
$131(14.15 \%)$
\end{tabular}

\begin{tabular}{ccc}
$\begin{array}{c}\text { Thyroiditis } \\
(n=294)\end{array}$ & & $\begin{array}{c}\text { Thyrotoxic crisis } \\
(n=11)\end{array}$ \\
\cline { 1 - 1 } $128(43.54 \%)$ & & 0 \\
$68(23.13 \%)$ & & $2(18.18 \%)$ \\
$4(1.70 \%)$ & & 0 \\
$6(2.04 \%)$ & & 0 \\
$24(8.16 \%)$ & & $2(18.18 \%)$ \\
$63(21.43 \%)$ & & $7(63.64 \%)$
\end{tabular}

$9 / 424(2.12 \%)$
$231 / 424(54.48 \%)$
$145 / 424(34.20 \%)$
$39 / 424(9.20 \%)$
$48 / 182(26.37 \%)$
$2 / 182(1.10 \%)$
$131 / 182(71.98 \%)$
$1 / 182(0.55 \%)$
$21 / 52(40.38 \%)$
$9 / 52(17.31 \%)$
$14 / 52(26.92 \%)$
$8 / 52(15.38 \%)$
$17 / 54(31.48 \%)$
$5 / 54(9.26 \%)$
$29 / 54(53.70 \%)$

$$
3 / 54(5.56 \%)
$$

$6 / 647(0.93 \%)$ $254 / 647(39.26 \%)$ $387 / 647(59.81 \%)$

$26 / 352(7.39 \%)$
$192 / 352(54.55 \%)$
$102 / 352(28.98 \%)$
$32 / 352(9.09 \%)$
$18 / 182(9.89 \%)$
$1 / 182(0.55 \%)$
$161 / 182(88.46 \%)$
$2 / 182(1.10 \%)$
$10 / 27(37.04 \%)$
$7 / 27(25.93 \%)$
$3 / 27(11.11 \%)$
$7 / 27(25.93 \%)$
$27 / 66(40.91 \%)$
$13 / 66(19.70 \%)$
$25 / 66(37.88 \%)$
$1 / 66(1.52 \%)$
$1 / 656(0.15 \%)$
$141 / 656(21.49 \%)$
$514 / 656(78.35 \%)$

$4 / 78(5.13 \%)$
$43 / 78(55.13 \%)$
$26 / 78(33.33 \%)$
$5 / 78(6.41 \%)$
$11 / 50(22.00 \%)$
0
$39 / 50(78.00 \%)$
0
$9 / 16(56.25 \%)$
$4 / 16(25.00 \%)$
$2 / 16(12.50 \%)$
$1 / 16(6.25 \%)$
$15 / 24(62.50 \%)$
$4 / 24(16.67 \%)$

$5 / 24(20.83 \%)$

0

$1 / 161(0.62 \%)$ $39 / 161(24.22 \%)$ $121 / 161(75.16 \%)$

0
0
$1 / 1(100.0 \%)$
0
0
0
$1(100.00 \%)$
0
0
0
0
0
0
$1 / 2(50.00 \%)$
0
$1 / 2(50.00 \%)$
0

$2 / 10(20.00 \%)$ $1 / 10(10.00 \%)$ $7 / 10(70.00 \%)$

${ }^{\dagger}$ Drug doses are reported in the VigiBase database in two ways, one is reported in $\mathrm{mg} / \mathrm{kg}$, the other is reported in total dose. Drug doses are grouped according to dosage and administration recommended in the drug prescription information. Recommended for Nivolumab: $3 \mathrm{mg} / \mathrm{kg}$ every 2 weeks or $240 \mathrm{mg}$ every 2 weeks or $480 \mathrm{mg}$ every 4 weeks for different cancers. Recommended for Pembrolizumab: $200 \mathrm{mg}$ every 3 weeks or $2 \mathrm{mg} / \mathrm{kg}$ for different cancers. Recommended for Ipilimumab: $3 \mathrm{mg} / \mathrm{kg}$ every 3 weeks or $10 \mathrm{mg} / \mathrm{kg}$ every 3 weeks for different cancers. Recommended for combined ICI therapy: Nivolumab $3 \mathrm{mg} / \mathrm{kg}$ followed by ipilimumab $1 \mathrm{mg} / \mathrm{kg}$ on the same day every 3 weeks for 4 doses, then Nivolumab $240 \mathrm{mg}$ every 2 weeks or $480 \mathrm{mg}$ every 4 weeks.

were patients treated with pembrolizumab, with time to onset of 72 days and 152 days, respectively.

Further analysis was conducted for follow-up reports after ICI-TD occurred. Only 1474 cases reported the final outcomes, of which most cases resulted in recovery or remission after standard treatment, but 10 cases $(0.68 \%)$ resulted in major or fatal consequences, including 6 cases of hypothyroidism, 2 cases of thyrotoxic crisis, 1 case of hyperthyroidism and 1 case of thyroiditis. Compared with ICI-associated hypothyroidism, there were more reports with recovery outcomes of ICI-associated hyperthyroidism and ICI-associated thyroiditis (387 (59.81\%) of 647 hypothyroidism patients; 514 (78.35\%) of 656 hyperthyroidism patients; 121 (75.16\%) of 161 thyroiditis patients; $\chi^{2}$ test for comparison between hypothyroidism and hyperthyroidism, $P<0.0001$; comparison between hypothyroidism and thyroiditis, $P=0.002$ ). Of all 10 thyrotoxic crisis cases that reported final outcomes, 7 were recovered, while 2 had major or fatal consequences (Table 4). 


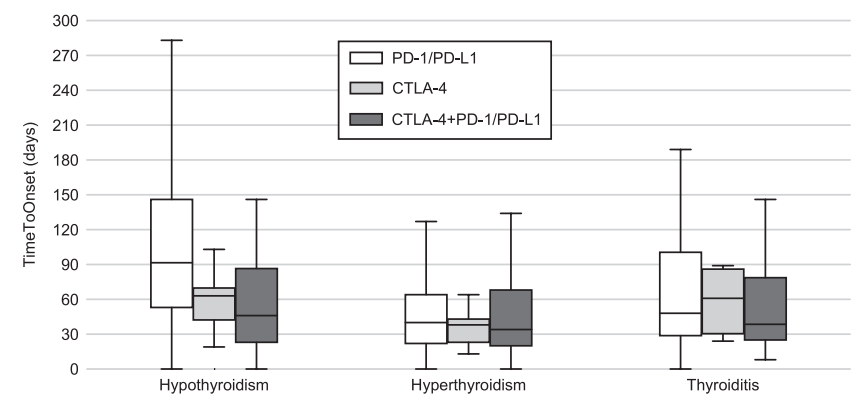

\section{Figure 1}

Time to onset for $\mathrm{ICl}$-associated thyroid dysfunctions (ICI-TDS). ICI-TDs are hypothyroidism ( $n=552)$, hyperthyroidism $(n=561)$, thyroiditis $(n=143)$ reported in Vigibase (Jan 1, 2011 to Mar 6, 2019). Among them, 136 cases of hyperthyroidism occurred followed by hypothyroidism, also referred to as transient thyrotoxicosis, and the mean duration of thyrotoxicosis was 36 days (IQR 9-63).

\section{Discussion}

To the best of our knowledge, we reported the largest and most extensive set of clinical characteristics of immune checkpoint inhibitor-associated thyroid dysfunction (ICI-TD) to date by analyzing individual case safety reports (ICSRs) from the WHO Global Database. Our study showed that ICI-associated hypothyroidism, hyperthyroidism, and thyroiditis were significantly increased, and the risk of receiving ICI combination therapy may be higher. We also reported 11 rare cases of thyrotoxic crisis.

ICIs have provided unprecedented efficacy for many cancer indications. However, since ICI blocks co-repressor molecules on $\mathrm{T}$ cells and other immune cells, and its release mediates tumour killing, ICI can also destroy the maintenance of immune homeostasis. Compared to chemotherapy, ICI has different toxicities and side effects, especially autoimmune manifestations of various organ systems including endocrine glands, which are commonly referred to as immune-related adverse events (irAEs). More and more endocrine irAEs for ICI treatment have been reported, including hypophysitis, thyroid dysfunction, insulin-deficient diabetes, and primary adrenal insufficiency (14). This study found that the incidence of ICI-TD has increased significantly over the past three years, especially in 2018, with hypothyroidism, hyperthyroidism and thyrotoxic crisis reports increased by nearly $50 \%$.

Previous studies have shown that hypophysitis is closely related to anti-CTLA-4 therapy, and thyroid dysfunction is closely related to anti-PD-1 therapy (2,
$15,16)$. This study observed that anti-PD therapy may have a higher risk of developing thyroid dysfunction than anti-CTLA-4 therapy, including hypothyroidism, hyperthyroidism, and thyroiditis. Combined ICIs therapy provides greater efficacy in many cancer therapies, and anti-PD-1 nivolumab with anti-CTLA-4 ipilimumab is the first approved ICI combination therapy. However, an increase in efficacy appears to result in higher frequency and more severe adverse drug reactions. Consistent with previous scientific literature reports $(17,18)$, our study indicated that of all these four ICI-TDs, ICI combination therapies may have a higher risk than monotherapy, especially for thyrotoxic crisis, with reporting odds ratio and 95\% CI values of 11.45 (2.44-53.84).

Most of our reports came from patients with lung cancer and melanoma. Among all analyzed ICI-TDs, the number of male cases was larger than the number of females. This seems to be inconsistent with the incidence of autoimmune thyroid disease in women. Whether the reason is related to the total cardinality of male cancer patients remains to be further studied. Most ICI-TDs occur within 3 months, and irAEs of combination therapy occur earlier than monotherapy. At the same time, we also found that anti-CTLA-4 therapy had a significantly earlier onset time of hypothyroidism than anti-PD therapy, but the onset times of hyperthyroidism associated with these two treatments were close. In addition, some hyperthyroidism cases can progress to hypothyroidism (19), which is closely related to anti-thyroid antibodies. A total of 136 cases of transient thyrotoxicosis were reported in our study. The median duration of thyrotoxicosis was 36 days (IQR 9-63), while a small-scale clinical trial indicated that the duration was 42 days (20). Because thyrotoxicosis lasts for a short time and is often overlooked clinically, the number of cases reported may be underestimated.

Importantly, this study analyzed ICI-associated thyrotoxic crisis, which was only reported as case reports in the past (21). Our report analysed 11 cases of thyrotoxic crisis, all of which were malignant melanoma patients, and most occurred in ICI combination therapy (7 cases, $63.64 \%$ ). Because the risk of thyrotoxic crisis is very high and can even be life-threatening, thyroid function needs to be closely monitored in clinical trials, with special attention to ICI combined therapy. It is recommended that the frequency of this monitoring be once a week, especially within one month after the initiation of combination therapy. The risk factors for ICI-associated thyrotoxic crisis are unclear. Whether the patient's own immune abnormality or the use of certain drugs that affect immune homeostasis increases autoimmune disease 
incidence after ICI combination therapy still remains to be explored in future clinical trials.

The severity of immune-related endocrine events is usually grades $1-2$, and the incidence of adverse events in grades 3-4 does not exceed $2 \%$ (9). Our study observed that in all ICI-TDs, only $0.68 \%$ had major or fatal consequences, and most cases of thyroid dysfunction were recovered or resolved after standard treatment. It is worth noting that 7 cases of thyrotoxic crisis were recovered (only 10 cases reported final outcomes). There were more cases of recovery from hyperthyroidism and thyroiditis than hypothyroidism, which may be due to the disease evolution of ICI-associated thyroid dysfunction. A typical patient using ICI who becomes thyrotoxic due to destructive thyroiditis, then develops hypothyroidism, is treated with levothyroxine. Previous reports have also indicated that patients with ICI-associated thyroiditis have a higher rate of tumour response and a longer progressionfree survival than those without thyroiditis (4). In general, ICI-TDs are usually controlled by standard treatment, with most cases recovering, and there is usually no need to reduce or discontinue drugs under close monitoring of thyroid function $(22,23)$.

We need to fully understand some of the limitations when using VigiBase. First, like all pharmacovigilance reporting databases, VigiBase pharmacovigilance analysis allows for signal monitoring to generate ideal reproducible prospective research hypotheses rather than validating hypotheses. Second, cases of thyroid disease caused by ICIs may not have been reported to the State Food and Drug Administration and therefore not submitted to VigiBase. However, compared with other smaller drug alert databases, such as FDA (AE Reporting System databases, AERs), European Medicine Agency (EMA), GSK company databases (OCEANS system), the bias of the proportional imbalance analysis of VigiBase is smaller due to overlap between these databases for adverse drug reactions. Third, the number of reports of different ICIs may be affected by other factors such as the degree of use of the drug, promotion, efficacy, and competitive bias. Fourth, the information component value does not imply a causal relationship between the target drug and the potential adverse effects caused but rather shows the quantitative dependence of ADR and drug. In general, a full understanding of the limitations of VigiBase can help us better analyze adverse reactions to make reasonable explanations and generate useful clinical research hypotheses.

VigiBasehasitslimitations, butspontaneousnotification is still the basis for assessing ADRs. The disproportionality analysis of ADRs in the pharmacovigilance database is an important method for drug safety research and postmarketing drug monitoring. Here, we use this method to identify thyroid irAEs. In addition, another clinically uncommon but significant complication (thyrotoxic crisis) was found in this analysis. Although the incidence rate of these thyroid irAEs is not available in VigiBase, some data and conclusions are instructive. As the use of ICI increases, timely diagnosis and treatment of drug-induced thyroid diseases will become much more important.

In summary, our study indicates that ICI-associated hypothyroidism, hyperthyroidism and thyroiditis cases increased significantly, and several reports of thyrotoxic crisis was observed. Most thyroid irAEs emerge early in the treatment process. Raising awareness of ICI-associated thyroid dysfunction is essential to improve the treatment of thyroid disease.

\section{Declaration of interest}

The authors declare that there is no conflict of interest that could be perceived as prejudicing the impartiality of this study.

\section{Funding}

This research did not receive any specific grant from any funding agency in the public, commercial or not-for-profit sector.

\section{Author contribution statement}

$X \mathrm{~L}, \mathrm{X} B$, and $\mathrm{X} C$ conceived the study, interpreted the data and drafted and critically revised the report. All authors of this paper have directly participated in the planning, review, data analysis and writing of this manuscript, and all authors approved the final version submitted.

\section{Acknowledgements}

The supplied data comes from various sources from VigiBase, and the probability that the suspected adverse effect is drug-related is not the same in all cases. The information does not represent the opinion of the UMC or the World Health Organization. Camilla Westerberg of the custom search team at the Uppsala Monitoring Centre (Uppsala, Sweden) research department is thanked for his help with the data extraction.

\section{References}

1 Bourke JM, O'Sullivan M \& Khattak MA. Management of adverse events related to new cancer immunotherapy (immune checkpoint inhibitors). Medical Journal of Australia 2016205 418-424. (https:// doi.org/10.5694/mja16.00586)

2 Ferrari SM, Fallahi P, Elia G, Ragusa F, Ruffilli I, Patrizio A, Galdiero MR, Baldini E, Ulisse S, Marone G et al. Autoimmune endocrine dysfunctions associated with cancer immunotherapies. International Journal of Molecular Sciences 201920 E2560. (https://doi. org/10.3390/ijms20102560)

3 Ryder M, Callahan M, Postow MA, Wolchok J \& Fagin JA. Endocrinerelated adverse events following ipilimumab in patients with advanced melanoma: a comprehensive retrospective review from a single institution. Endocrine-Related Cancer 201421 371-381. (https:// doi.org/10.1530/ERC-13-0499) 
4 Lei M, Michael A, Patel S \& Wang D. Evaluation of the impact of thyroiditis development in patients receiving immunotherapy with programmed cell death-1 inhibitors. Journal of Oncology Pharmacy Practice 201925 1402-1411. (https://doi. org/10.1177/1078155219829813)

5 Yamauchi I, Sakane Y, Fukuda Y, Fujii T, Taura D, Hirata M, Hirota K, Ueda Y, Kanai Y, Yamashita Y et al. Clinical features of Nivolumabinduced thyroiditis: a case series study. Thyroid 201727 894-901. (https://doi.org/10.1089/thy.2016.0562)

6 Yonezaki K, Kobayashi T, Imachi H, Yoshimoto T, Kikuchi F, Fukunaga K, Sato S, Ibata T, Yamaji N, Lyu J et al. Combination therapy of ipilimumab and nivolumab induced thyroid storm in a patient with Hashimoto's disease and diabetes mellitus: a case report. Journal of Medical Case Reports 201812 171. (https://doi.org/10.1186/ s13256-018-1708-x)

7 McMillen B, Dhillon MS \& Yong-Yow S. A rare case of thyroid storm. BMJ Case Reports 20162016 bcr2016214603. (https://doi. org/10.1136/bcr-2016-214603)

8 Sznol M, Postow MA, Davies MJ, Pavlick AC, Plimack ER, Shaheen M, Veloski C \& Robert C. Endocrine-related adverse events associated with immune checkpoint blockade and expert insights on their management. Cancer Treatment Reviews 201758 70-76. (https://doi. org/10.1016/j.ctrv.2017.06.002)

9 Eigentler TK, Hassel JC, Berking C, Aberle J, Bachmann O, Grünwald V, Kähler KC, Loquai C, Reinmuth N, Steins M et al. Diagnosis, monitoring and management of immune-related adverse drug reactions of anti-PD-1 antibody therapy. Cancer Treatment Reviews 201645 7-18. (https://doi.org/10.1016/j.ctrv.2016.02.003)

10 Lindquist M. VigiBase, the WHO Global ICSR Database System: basic facts. Drug Information Journal 200842 409-419. (https://doi. org/10.1177/009286150804200501)

11 Ang PS, Chen Z, Chan CL \& Tai BC. Data mining spontaneous adverse drug event reports for safety signals in Singapore - a comparison of three different disproportionality measures. Expert Opinion on Drug Safety 201615 583-590. (https://doi.org/10.1517/14 740338.2016.1167184)

12 Bate A, Lindquist M, Edwards IR, Olsson S, Orre R, Lansner A \& De Freitas RM. A bayesian neural network method for adverse drug reaction signal generation. European Journal of Clinical Pharmacology 199854 315-321. (https://doi.org/10.1007/s002280050466)

13 Norén GN, Hopstadius J \& Bate A. Shrinkage observed-to-expected ratios for robust and transparent large-scale pattern discovery. Statistical Methods in Medical Research 201322 57-69. (https://doi. org/10.1177/0962280211403604)
14 Chang LS, Barroso-Sousa R, Tolaney SM, Hodi FS, Kaiser UB \& Min L. Endocrine toxicity of cancer immunotherapy targeting immune checkpoints. Endocrine Reviews 201940 17-65. (https://doi. org/10.1210/er.2018-00006)

15 Khoja L, Day D, Wei-Wu Chen T, Siu LL \& Hansen AR. Tumour- and class-specific patterns of immune-related adverse events of immune checkpoint inhibitors: a systematic review. Annals of Oncology 2017 28 2377-2385. (https://doi.org/10.1093/annonc/mdx286)

16 Byun DJ, Wolchok JD, Rosenberg LM \& Girotra M. Cancer immunotherapy - immune checkpoint blockade and associated endocrinopathies. Nature Reviews: Endocrinology 201713 195-207. (https://doi.org/10.1038/nrendo.2016.205)

17 Scott ES, Long GV, Guminski A, Clifton-Bligh RJ, Menzies AM \& Tsang VH. The spectrum, incidence, kinetics and management of endocrinopathies with immune checkpoint inhibitors for metastatic melanoma. European Journal of Endocrinology 2018178 173-180. (https://doi.org/10.1530/EJE-17-0810)

18 Barroso-Sousa R, Barry WT, Garrido-Castro AC, Hodi FS, Min L, Krop IE \& Tolaney SM. Incidence of endocrine dysfunction following the use of different immune checkpoint inhibitor regimens: a systematic review and meta-analysis. JAMA Oncology 20184 173-182. (https://doi.org/10.1001/jamaoncol.2017.3064)

19 Iyer PC, Cabanillas ME, Waguespack SG, Hu MI, Thosani S, Lavis VR, Busaidy NL, Subudhi SK, Diab A \& Dadu R. Immune-related thyroiditis with immune checkpoint inhibitors. Thyroid 201828 1243-1251. (https://doi.org/10.1089/thy.2018.0116)

20 Lee H, Hodi FS, Giobbie-Hurder A, Ott PA, Buchbinder EI, Haq R, Tolaney S, Barroso-Sousa R, Zhang K, Donahue H et al. Characterization of thyroid disorders in patients receiving immune checkpoint inhibition therapy. Cancer Immunology Research 20175 1133-1140. (https://doi.org/10.1158/2326-6066.CIR-17-0208)

$21 \mathrm{Yu}$ C, Chopra IJ \& Ha E. A novel melanoma therapy stirs up a storm: ipilimumab-induced thyrotoxicosis. Endocrinology, Diabetes and Metabolism Case Reports 20152015 140092. (https://doi.org/10.1530/ EDM-14-0092)

22 Johnson DB, Sullivan RJ, Ott PA, Carlino MS, Khushalani NI, Ye F, Guminski A, Puzanov I, Lawrence DP, Buchbinder EI et al. Ipilimumab therapy in patients with advanced melanoma and preexisting autoimmune disorders. JAMA Oncology 20162 234-240. (https://doi.org/10.1001/jamaoncol.2015.4368)

23 Torino F, Barnabei A, Paragliola R, Baldelli R, Appetecchia M \& Corsello SM. Thyroid dysfunction as an unintended side effect of anticancer drugs. Thyroid 201323 1345-1366. (https://doi. org/10.1089/thy.2013.0241)

Received 12 July 2019

Revised version received 22 October 2019

Accepted 24 October 2019 